\title{
Rapport de gestion électronique de la FMH: un atout à tous les niveaux
}

\author{
Anne-Geneviève Bütikofer
}

lic. iur., secrétaire générale de la FMH

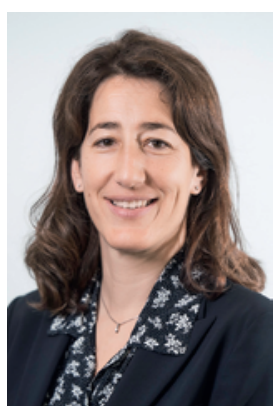

L'avènement des technologies, bien qu'il constitue un progrès indéniable pour le monde du travail, n'en allège pas pour autant la charge, bien au contraire. Il est donc important d'offrir des outils pratiques, adaptés, conviviaux et dont l'utilisation n'engendre pas un surcroît de travail à son utilisateur.

L’année passée, la version papier du rapport de gestion était supprimée et remplacée par une seule et unique version ePaper qui se présentait selon la même mise en page qu'auparavant mais sur un support électronique. Je vous annonçais que cette forme n'était que transitoire, avant que nous ne mettions à disposition notre rapport de gestion électronique en ligne dans sa version définitive et interactive sur notre site internet www.fmh.ch.

\section{La lecture de l'utilisateur est ponctuée non} seulement d'infographies attractives et interactives, mais également d'animations vidéo.

Le nouveau rapport de gestion électronique, tel qu'il a été souhaité par la Chambre médicale, est dès aujourd'hui accessible au lien suivant: www.report2016. fmh.ch. Si cette nouvelle forme de publication d'une part permet d'optimiser les coûts de production, elle s'inscrit d'autre part à la fois dans une démarche de développement durable respectueuse de l'environnement mais également dans une logique d'adaptation à l'usage des lecteurs, respectivement aux nouvelles technologies de communication. En effet ce rapport de gestion est désormais accessible où que vous vous trouviez, quel que soit le support que vous utilisiez pour le consulter, ordinateur, ordinateur portable, tablette ou téléphone portable. Le contenu multimédia a été repensé et la lecture de l'utilisateur est ponctuée non seulement d'infographies attractives et interactives, mais également d'animations vidéo. Ce numéro offre notamment de brèves prises de position filmées des acteurs de la santé suivants, la Dresse Anja Zyska Cherix, Vice-présidente de l'ASMAC, Marcel Altherr, Head of Interdisciplinary Program «Data Worlds», University of Applied Sciences and Arts,
Lucerne, ainsi que le Président de la FMH, Dr méd. Jürg Schlup, qui donnent entre autres leur vision de l'optimisation du système de santé et de l'évolution de la profession de médecin.

La navigation dans le rapport est facilitée grâce à un contraste visuel associé à de grands titres, des lignes de séparation claires, des chiffres clés ou encore des citations personnelles. La recherche d'éléments au travers de la fonction recherche sur toutes les pages du rapport est aisée. Le lecteur peut de manière interactive partager certaines pages du rapport ou le rapport intégral via les médias sociaux. Le rapport ou les chapitres qui vous intéressent le plus peuvent également être téléchargés, imprimés et conservés sous format PDF. Enfin, de multiples liens vers le site de la FMH permettent d'obtenir des informations plus détaillées et actuelles sur un domaine d'activité ou un projet spécifique.

Vous retrouverez par ailleurs toutes les informations utiles qui vous permettent de retracer les activités de la FMH en 2016 au travers des rapports du Comité central et des divisions du Secrétariat général de la FMH, mais également des comptes annuels et du rapport de situation tel qu'exigé par la loi.

Trois acteurs de la santé présentent leur vision de l'optimisation du système de santé et de l'évolution de la profession de médecin.

Alors que la charge administrative continue d'augmenter de manière inquiétante pour les médecins, le Secrétariat général de la FMH s'efforce, au niveau opérationnel, d'alléger le travail de nos membres dans la mesure du possible, notamment en facilitant l'accès à l'information. Cette nouvelle prestation attractive et moderne est une étape supplémentaire dans la modernisation et l'adaptation des plateformes de communication de la FMH, qu'il s'agisse du site Internet ou d'Intranet soit de la plateforme des membres myFMH. J'espère dès lors que les utilisateurs fassent le même constat et en apprécient la lecture, et vous encourage à prendre connaissance de ce rapport sous www.report2016.fmh.ch. 